\title{
Cricopharyngeal myotomy with mucosal repair: Long term (5 year) outcomes following surgery
}

\author{
Mr Jonathan Bird, ENT Registrar \\ Miss Elinor Warner, ENT Registrar \\ Mr Rogan Corbridge, Consultant Otolaryngologist \\ Royal Berkshire Hospital, Reading, United Kingdom.
}

\section{Introduction}

Cricopharyngeal myotomy (CPM )is an established technique for management of dysphagia secondary to failure of relaxation of the upper oesophageal sphincter which is largely made up of the cricopharyngeus muscle. Various techniques have been described to manage cricopharyngeal spasm including endoscopic approaches for botulinum injection, dilation, endoscopic CPM and open an approach CPM. Endoscopic laser CPM is associated with reduced operative times, inpatient stay and lower morbidity ${ }^{1}$. Although efficacy of cricopharyngeal myotomy with mucosal repair (CPM-MR) techniques are established in the short term², long term outcomes of these patients and indeed the implications of the surgery still require evaluation. To date the national institute of health and clinical excellence (NICE) guidelines state that although recognised as a technique more long-term data is required before a formal recommendation regarding endoscopic laser CPM-MR can be issued ${ }^{3}$.

\section{Methods}

Using our local CPM database, patients were identified who had had laser CPM-MR over five years previously.

All subjects who had been offered surgery due had symptoms of dysphagia with evidence of cricopharyngeal hypertrophy on barium swallow, were fit medically fit for a general anesthetic and had adequate endoscopic access to the cricopharyngeus. These patients received a telephone follow up from the authors (JB \& EW) in August 2017 and a repeat SSQ and RSI score was obtained and then compared to scores given preoperatively and acutely post-operatively.

\section{Results}

15 patients were identified who fulfilled the criteria of having had a previous CPM with mucosal repair five years previously with a pre-operative and acute post-operative SSQ and RSI score. Three patients had passed away since the time of surgery due to unrelated health reasons. Two patients were uncontactable, therefore we identified ten patients who could be re-assessed.

No patient declined to partake (response rate of $100 \%$ ). The average age of the ten patients at operation was 70.5 years (62-84 years) consisting of seven males and three females. The mean follow up time from primary surgery was 66 months (6079 months). No patient had required revision surgery or suffered a complication of surgery. The results of pre-operative scores, post-operative scores and scores as of August 2017 are summarised in Charts 1 and 2.

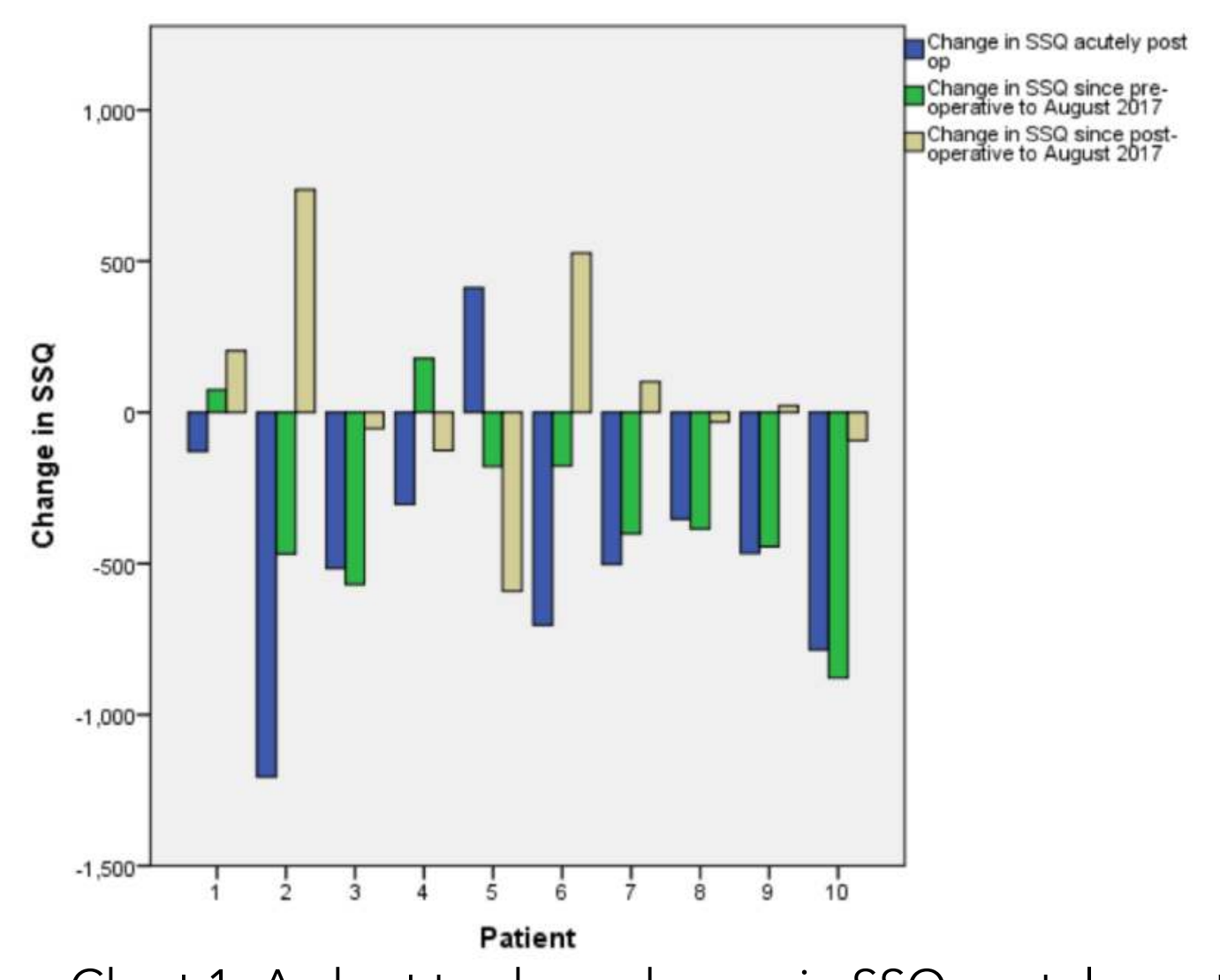

Chart 1: A chart to show change in SSO acutely post operatively, pre-operatively versus August 2017 and acutely post-operative to August 2017.

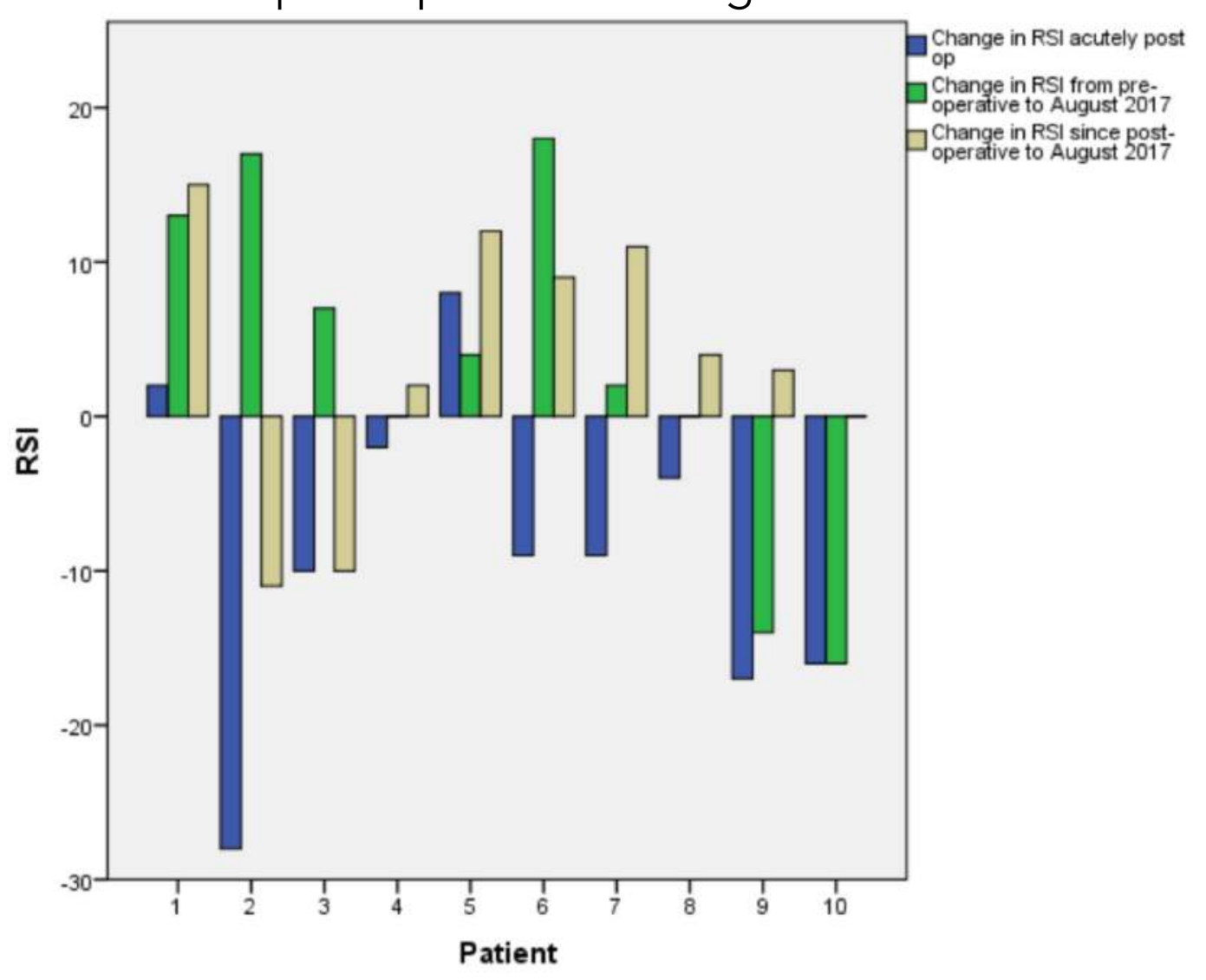

Chart 2: A chart to show change in RSI acutely post operatively, pre-operatively versus August 2017 and acutely post-operative to August 2017.

\section{Results (continued)}

With regards to the $S S Q, 90 \%(n=9)$ had a reduction in symptoms in the acute period and $90 \%(n=9)$ have a better SSQ score in August 2017 than they did pre-operatively. Interestingly the one patient who had an acutely worse SSQ (patient 5) acutely now reports symptoms which are better than pre-operatively. Subjectively $60 \%(n=6)$ of patients have had a deterioration in their SSQ score over the following five-year period since their operation with one patient (patient 1) now more symptomatic than before their laser CPM-MR. The RSI scores demonstrated that CPM acutely reduced reflux symptoms in $80 \%(n=8)$ when surveyed but over the subsequent five-year period since their operation the RSI is now worse or the same than pre-operatively in $70 \%(n=7)$.

- In our cohort of patients, the majority had a subjectively improved swallow in the long-term although some did have regression of symptoms but remained better than pre-operatively.

- Subjectively our cohort of patients appeared to favour less well in the long-term with regards to reflux symptoms, the majority fairing worse than pre-operatively at least 5 years previously.

- This research would suggest that as clinicians we can advise patients that in the long-term, laser cricopharyngeal myotomy will improve swallowing symptoms but that their reflux symptoms may be exacerbated over time. 